\title{
Current on and Input Impedance of a Cylindrical Antenna *
}

\author{
Yung Ming Chen and Joseph B. Keller
}

\author{
Contribution from Institute of Mathematical Sciences, New York University, New York, N.Y.
}

(Received March 13, 1961; revised July 27, 1961.)

\begin{abstract}
The electric current on a finite antenna is expressed as the sum of a current emanating from the gap and two currents reflected from the ends. These currents are determined for a perfectly conducting hollow pipe of circular-cross section. The antenna is excited by an electric field parallel to the axis applied across a gap of finite width which encircles the antenna. The currents are also determined for a thin antenna of any cross section. From the results the current on and the input admittance of the antennas are determined. It is shown that the thin antenna theory yields an incorrect result for the admittance because it ignores a boundary layer effect near the gap.
\end{abstract}

\section{Introduction}

The fundamental problem in the analysis of a transmitting antenna is that of determining the electromagnetic field everywhere in space which results when a prescribed input is supplied to the antenna. From this field all the properties of the antenna can be found. One of the most important of these properties is the distribution of current in the antenna, or on its surface if the antenna is a perfect conductor. Knowledge of this current is essentially equivalent to knowledge of the field since either can be found from the other. Most analyses of transmitting antennas are devoted to the determination of the current, which is the more convenient to deal with. We shall follow this practice in determining the current on a cylindrical antenna of finite or infinite length.

A basic difficulty arises in introducing the source of the field into a transmitting antenna problem. The difficulty is that the electromagnetic field produced by the source is affected by the antenna. In principle it is necessary to determine this field simultaneously with that produced by the antenna. However, in order to render the analysis feasible, it is customary to split this problem in two - an antenna problem and a feed problem. In the antenna problem the feed is idealized by assuming that it produced a specified applied field across a gap or aperture in the antenna surface. In the feed problem the antenna and the space or medium surrounding it are idealized by assuming that they present a certain terminal load impedance to the waveguide or transmission line from the source to the antenna. In this procedure, which we shall follow, it is necessary to determine the impedance just referred to. It is called the input impedance of the antenna, and can be found from the antenna current in the antenna problem.

It has been recognized generally that the decomposition of the total electromagnetic problem into an

*The research in this paper was partially supported by the Office of Naval Research under Contract No. 285(48). antenna problem and a feed problem introduces inaccuracies. In particular, properties of the solution of the antenna problem which depend upon details of the gap size or shape, or of the applied field distribution across it, are considered to be irrelevant in applying the theory to actual antennas. Therefore the gap is often taken to have zero width, since details which depend upon the width are assumed to be unimportant. Unfortunately this simplification introduces a technical difficulty into the analysis; namely the antenna current at the gap is then infinite. The input admittance of the antenna, which is determined by this current, is also infinite. Since only the component of current out of phase with the applied voltage is infinite, it is only the input susceptance which is infinite, while the input conductance is finite.

We avoid this difficulty by keeping the gap of finite width, and thus we obtain a finite input admittance. We also suggest a method for applying the results to actual antennas by subtracting the gap-dependent input susceptance from our result and replacing it by the input susceptance of the actual gap or feed.

The singularity in the current at the gap and the consequent infinite value of the input susceptance have been pointed out by various authors. However they have been overlooked by many others who have concerned themselves with the theory of thin antennas. (A thin antenna is one in which two dimensions, called transverse, are small compared to the third, called longitudinal, and to the wavelength of the radiated field.) The current on a thin antenna is usually obtained by solving an integral equation, which is an approximation to the exact integral equation satisfied by the exact current. It can also be obtained by approximating the expression for the exact current in cases where the exact current can be found. Both of these methods lead to an expression for the current on a thin antenna which is finite at the gap, even though the gap is of zero width. But, as we shall see, this expression for the 
current is not valid at or near the gap. Instead there is a narrow region in which the current decreases from its infinite value at the gap to the finite value given by the thin antenna theory. This is an instance of a boundary layer effect, such as often arises in the asymptotic expansion of solutions of boundary value problems. As a consequence the values of the input admittance given by the usual thin antenna theories, are incorrect.

The essence of our analysis of finite antennas, presented in section 2 , is to represent the current in terms of a current emanating from the gap and two currents reflected from the ends. We identify these currents as the solutions of simpler problems for infinite and semi-infinite antennas respectively. In section 3 we determine the current on an infinite cylindrical antenna of circular cross section, or on a thin cylindrical antenna of any cross section. In sections 4 and 5 we evaluate these currents both near the gap and far from it. In section 6 we analyze the current reflected from the end of a semi-infinite cylindrical antenna of circular cross section, and of a thin antenna of any cross section. Finally in section 7 we use the results of sections 3 to 6 in the theory of section 2 to determine the current on, and input admittance of, a finite cylindrical antenna.

Many of the results which we use in our analysis have been obtained before by others and we shall point this out where it seems appropriate. The approximations upon which our theory is based make it more accurate the longer the antenna. Therefore we shall not have occasion to consider short antennas. A comprehensive account of them is given in the book of R. W. P. King [1956].

\section{Current on a Finite Antenna}

Let us consider a straight or bent antenna of uniform or nonuniform cross section and let $z$ denote distance along the center line of the antenna. Suppose the ends of the antenna are at $z=-z_{1}$ and $z=z_{2}$ and that a gap extends from $z=-\delta$ to $z=\delta$. Let $V e^{i \omega t}$ be the voltage applied across the gap and let $V I(z) e^{i \omega t}$ be the total current excited on the outer surface of the antenna by this voltage. We wish to determine this current and from it the antenna's input admittance $Y=1 / 2[I(\delta)+I(-\delta)]$. Henceforth we shall omit the factor $e^{i \omega t}$.

We write the current as a sum of three terms

$$
I(z)=I_{\delta}(z)+B_{1} I_{1}\left(z_{1}+z\right)+B_{2} I_{2}\left(z_{2}-z\right) .
$$

We interpret $I_{\delta}(z)$ as the current emanating directly from the gap, $B_{1} I_{1}$ as the current reflected from the end $z=-z_{1}$ and $B_{2} I_{2}$ as the current reflected from the end $z=z_{2}$. For thin antennas, similar ways of writing the current have been suggested and used by various authors, especially E. Hallen [1948] and L. A. Vainshtein [1959 a, b]. More precisely we define $I_{\delta}(z)$ to be the current which the same voltage across the same gap would produce on an infinitely long antenna $\left(z_{1}=z_{2}=\infty\right)$. This definition is unambiguous in the case of a straight antenna of uniform cross section but depends upon how the antenna is extended to infinity in other cases. We next define $I_{1}\left(z_{1}+z\right)$ to be the dimensionless current reflected from the end $z=-z_{1}$ when unit current is incident on that end and the other end of the antenna is at infinity $\left(z_{2}=\infty\right)$. We define $I_{2}\left(z_{2}-z\right)$ analogously.

On the basis of these definitions we can now determine the constants $B_{1}$ and $B_{2}$ in (1). The current incident on the end $z=-z_{1}$ is just $I_{\delta}\left(-z_{1}\right)$ $+B_{2} I_{2}\left(z_{2}+z_{1}\right)$ and this must equal $B_{1}$, since $I_{1}\left(z_{1}+z\right)$ is the current reflected for unit incident current. Thus we have

Similarly

$$
B_{1}=I_{\delta}\left(-z_{1}\right)+B_{2} I_{2}\left(z_{2}+z_{1}\right) .
$$

$$
B_{2}=I_{\delta}\left(z_{2}\right)+B_{1} I_{1}\left(z_{1}+z_{2}\right) .
$$

By solving these equations for $B_{1}$ and $B_{2}$ and inserting the results into (1), we obtain

$I(z)=I_{\delta}(z)+\frac{\left[\begin{array}{l}{\left[I_{\delta}\left(-z_{1}\right)+I_{\delta}\left(z_{2}\right) I_{2}\left(z_{1}+z_{2}\right)\right] I_{1}\left(z_{1}+z\right)} \\ +\left[I_{\delta}\left(z_{2}\right)+I_{\delta}\left(-z_{1}\right) I_{1}\left(z_{1}+z_{2}\right)\right] I_{2}\left(z_{2}-z\right)\end{array}\right] .}{1-I_{1}\left(z_{1}+z_{2}\right) I_{2}\left(z_{1}+z_{2}\right)}$.

It is interesting to note that (2) and (3) have a nontrivial solution for $B_{1}$ and $B_{2}$ in the absence of the exciting current $I_{\delta}(z)$ when the determinant of the coefficients vanishes.

$$
1-I_{1}\left(z_{1}+z_{2}\right) I_{2}\left(z_{1}+z_{2}\right)=0 .
$$

This equation determines the values of $\omega$ at which there can be unexcited or free currents on the antenna. The current in any such mode is from (1) and (3), proportional to $I_{1}\left(z_{1}+z\right)+I_{1}\left(z_{1}+z_{2}\right) I_{2}\left(z_{2}-z\right)$.

From (4) we can compute the input admittance $Y$. If we neglect $\delta$ compared to $z_{1}$ and $z_{2}$ which is usually permissible in practice, the result simplifies to

$$
\begin{aligned}
Y=\frac{1}{2}\left[I_{\delta}(\delta)+I_{\delta}(-\delta)\right] \\
+\frac{\left[\begin{array}{l}
\left.I_{\delta}\left(-z_{1}\right)+I_{\delta}\left(z_{2}\right) I_{2}\left(z_{1}+z_{2}\right)\right] I_{1}\left(z_{1}\right) \\
+\left[I_{\delta}\left(z_{2}\right)+I_{\delta}\left(-z_{1}\right) I_{1}\left(z_{1}+z_{2}\right)\right] I_{2}\left(z_{2}\right)
\end{array}\right] .}{1-I_{1}\left(z_{1}+z_{2}\right) I_{2}\left(z_{1}+z_{2}\right)}
\end{aligned}
$$

For a symmetrical antenna $I_{\delta}(-z)=I_{\delta}(z), z_{1}=z_{2}$ and $I_{1}=I_{2}$. Then (4) and (6) simplify to

$$
\begin{gathered}
I(z)=I_{\delta}(z)+\frac{I_{\delta}\left(z_{1}\right)}{1-I_{1}\left(2 z_{1}\right)}\left[I_{1}\left(z_{1}+z\right)+I_{1}\left(z_{1}-z\right)\right] \\
Y=I_{\delta}(\delta)+\frac{I_{\delta}\left(z_{1}\right) I_{1}\left(z_{1}\right) .}{1-I_{1}\left(2 z_{1}\right)} .
\end{gathered}
$$

The resonance condition (5) becomes $I_{1}\left(2 z_{1}\right)= \pm 1$, but only $I_{1}\left(2 z_{1}\right)=1$ occurs as a resonance denominator in (7) and (8). This is because center feeding can excite only the symmetric modes, and not the antisymmetric ones corresponding to $I_{1}\left(2 z_{1}\right)=-1$. 
For a cylindrical antenna we assume that away from the ends $I_{1}(z)$ and $I_{2}(z)$ vary with $z$ in the same manner as does $I_{\delta}(z)$. Therefore we write

$$
I_{1}(z)=I_{2}(z)=C I_{\delta}(z) .
$$

The constant $C$ in (9) depends upon the shape and size of the end of the antenna. We shall determine it in section 6 for a hollow cylindrical antenna of circular cross section and for a thin cylindrical antenna of any cross section. By means of (9) the results (4) to (8) can be simplified so that they involve only the one function $I_{\delta}(z)$ and the constant $C$. Thus, for example, (7) and (8) which apply to a symmetric antenna become, when $z$ is away from the ends $\pm \tilde{z}_{1}$,

$$
\begin{gathered}
I(z)=I_{\delta}(z)+\frac{C I_{\delta}\left(z_{1}\right)}{1-C I_{\delta}\left(2 z_{1}\right)}\left[I_{\delta}\left(z_{1}+z\right)+I_{\delta}\left(z_{1}-z\right)\right] \\
Y=I_{\delta}(\delta)+\frac{C I_{\delta}^{2}\left(z_{1}\right)}{1-C I_{\delta}\left(2 z_{1}\right)} .
\end{gathered}
$$

We observe that the input admittance of a finite antenna is expressed by (6), (8), and (11) as the sum of the input admittance of an infinite antenna, $\frac{1}{2}\left[I_{\delta}(\delta)+I_{\delta}(-\delta)\right]$, and a finite additional term. In section 4 we shall show that $I_{\delta}( \pm \delta)$ is finite for $\delta>0$, in the case of the circular cylindrical antenna, and that it becomes infinite as $\delta$ goes to zero. It is only the imaginary part of $I_{\delta}( \pm \delta)$, and thus only the susceptance of the antenna, that becomes infinite. This is due to the infinitely large capacitance of the gap of zero width, as is well known, and as has been verified by $\mathrm{T}$. T. Wu and R. W. P. King [1959].

Since for small values of $\delta$ the susceptance depends critically on $\delta$, it might be inappropriate to use the above theoretical results for an actual antenna with a feed which is different from the gap of width $2 \delta$. We can overcome this difficulty by subtracting from the theoretical value of the admittance of a finite antenna the susceptance of the corresponding infinite antenna. Then we can add to this difference the susceptance of the feed of the actual antenna. In this way the theoretical results can be used in practice. A similar procedure was suggested by Wu and King [1959].

\section{Current on an Infinite Antenna}

We shall now consider a linear antenna consisting of an infinitely long perfectly conducting thin hollow pipe of circular cross section and radius $a$. In a cylindrical coordinate system $(r, \theta, z)$ let the pipe lie in the surface $r=a,|z|>\delta$. In the gap we assume that the applied electric field is

$$
E_{z}^{\text {app lied }}=-\frac{V}{2 \delta} e^{i \omega t}, \quad r=a,|z|>\delta .
$$

On the pipe

$$
E_{z}=0 \quad r=a,|z|>\delta .
$$

We seek the electromagnetic field throughout space, assuming that it vanishes at infinity, or else satisfies an appropriate radiation condition if the conductivity of the surrounding medium is zero.

This problem has been solved by various authors, so it should suffice to indicate the method of solution and to present the main formulas. First we note that as a consequence of Maxwell's equations, $E_{z}$ satisfies

$$
\left(\Delta+k^{2}\right) E_{z}=0 .
$$

Here $k=\left(\epsilon \mu \omega^{2}-i \sigma \mu \omega\right)^{1 / 2}$ is the propagation constant of the field while $\epsilon, \mu$, and $\sigma$ are the dielectric constant, permeability, and conductivity of the medium surrounding the antenna.

The radiating solution of (12) to (14) is easily found by separation of variables and is

$$
\begin{array}{r}
E_{z}(r, z)=-\frac{V}{\pi} \int_{0}^{\infty} \frac{\sin \beta \delta}{\beta \delta} \frac{H_{0}^{(2)}\left(\left[k^{2}-\beta^{2}\right]^{1 / 2} r\right)}{H_{0}^{(2)}\left(\left[k^{2}-\beta^{2}\right]^{1 / 2} a\right)} \cos \beta z d \beta, \\
r \geqq a
\end{array}
$$

$E_{z}(r, z)=-\frac{V}{\pi} \int_{0}^{\infty} \frac{\sin \beta \delta}{\beta \delta} \frac{J_{0}\left(\left[k^{2}-\beta^{2}\right]^{1 / 2} r\right)}{J_{0}\left(\left[k^{2}-\beta^{2}\right]^{1 / 2} a\right)} \cos \beta z d \beta$, $r \leqq a$.

The expression (15) is contained in the results of J. A. Stratton and L. J. Chu [1941], S. A. Schelkunoff [1941], V. V. Vladimirskii [1944], E. Hallen [1948], and F. H. Northover [1958]. The magnetic field has only an angular component $H_{\theta}$ which is derivable from a vector potential with only one component $A_{z}$ by the relation $H_{\theta}=-\frac{1}{\mu} \frac{\partial}{\partial r} A_{z}$. The potential $A_{z}$ is related to $E_{z}$ by the equation

$$
\left(\frac{\partial^{2}}{\partial z^{2}}+k^{2}\right) A_{z}=i \omega \epsilon \mu E_{z}
$$

The outgoing solution of (17) is

$A_{z}=-i V \omega \mu \epsilon \pi^{-1}$

$\times \int_{0}^{\infty} \frac{\sin \beta \delta}{\beta \delta} \frac{H_{0}^{(2)}\left(\left[k^{2}-\beta^{2}\right]^{1 / 2} r\right)}{H_{0}^{(2)}\left(\left[k^{2}-\beta^{2}\right]^{1 / 2} a\right)} \frac{\cos \beta z}{\left(k^{2}-\beta^{2}\right)} d \beta \quad r \geqq a$

$A_{z}=-i V \omega \mu \epsilon \pi^{-1}$

$\times \int_{0}^{\infty} \frac{\sin \beta \delta}{\beta \delta} \frac{J_{0}\left(\left[k^{2}-\beta^{2}\right]^{1 / 2} r\right)}{J_{0}\left(\left[k^{2}-\beta^{2}\right]^{1 / 2} a\right)} \frac{\cos \beta z}{\left(k^{2}-\beta^{2}\right)} d \beta \quad r \leqq a$.

The surface current density on either side of the antenna is given by $\vec{n} \times \vec{H}(a, z)$ where $\vec{n}$ is the unit outward normal to that side of the antenna surface; i.e., pointing inward on the inside and outward on the outside. Since $\vec{H}$ has only a $\theta$ component, the current has only a z component. We define the total currents, $V I_{\delta}^{\prime}(z)$ and $V I_{\delta}(z)$ on the inner and outer surfaces respectively, as the $z$ components of $2 \pi a \vec{n} \times \vec{H}(a, z)$. Then from (18), (19), and the 
relation of $H$ to $A_{z}$ we obtain

$$
\begin{aligned}
& I_{\delta}(z)=2 i a \omega \epsilon \delta^{-1} \\
& \int_{0}^{\infty} \frac{\sin \beta \delta \cos \beta z}{\beta\left(k^{2}-\beta^{2}\right)^{1 / 2}} \frac{H_{0}^{(2) \prime}\left(\left[k^{2}-\beta^{2}\right]^{1 / 2} a\right)}{H_{0}^{(2)}\left(\left[k^{2}-\beta^{2}\right]^{1 / 2} a\right)} d \beta \quad \\
& I_{\delta}^{\prime}(z)=-2 i a \omega \epsilon \delta^{-1} \int_{0}^{\infty} \frac{\sin \beta \delta \cos \beta z}{\beta\left(k^{2}-\beta^{2}\right)^{1 / 2}} \frac{J_{0}^{\prime}\left(\left[k^{2}-\beta^{2}\right]^{1 / 2} a\right)}{J_{0}\left(\left[k^{2}-\beta^{2}\right]^{1 / 2} a\right)} d \beta .
\end{aligned}
$$

Upon adding $I_{\delta}$ and $I_{\delta}^{\prime}$ we obtain the total current which becomes, upon making use of the Wronskian of the Bessel and Hankel functions in (20) and (21),

$$
\begin{aligned}
I_{\delta}(z)+I_{\delta}^{\prime}(z)=4 \omega \epsilon \pi^{-1} \int_{0}^{\infty} \frac{\sin \beta \delta}{\beta \delta} \\
\\
\quad \frac{\cos \beta z}{\left(k^{2}-\beta^{2}\right) J_{0}\left(\left[k^{2}-\beta^{2}\right]^{1 / 2} a\right) H_{0}^{(2)}\left(\left[k^{2}-\beta^{2}\right]^{1 / 2} a\right)} d \beta .
\end{aligned}
$$

These results have all been obtained by one or more of the authors referred to above. The total current (22) with $\delta=0$ was also obtained by T. T. Wu and R. W. P. King [1959] and R. H. Duncan and F. A. Hinchey [1960].

When $k a<<1$ the result (20) for $I_{\delta}(z)$ can be simplified to

$$
I_{\delta}(z) \approx i \omega \epsilon \int_{-\infty}^{\infty} \frac{\sin \beta \delta}{\beta \delta} \frac{e^{-i \beta|z|}}{\left(k^{2}-\beta^{2}\right)} \frac{d \beta}{\log \frac{\Gamma a\left(k^{2}-\beta^{2}\right)^{1 / 2}}{2}} .
$$

Here $\Gamma \approx 1.781$ is the exponential of Euler's constant. The expression (22) with $\delta=0$ is the usual result for a thin antenna with a gap of zero width. It is given by Vladimirskii [1944], Hallen [1956], and Vainshtein $[1959 \mathrm{a}, \mathrm{b}]$. If the cross section is not circular, $a$ denotes the equivalent radius. We shall see that this approximation is incorrect near the gap.

\section{The Current Near the Gap}

We shall now evaluate the currents $I_{\delta}(z)$ and $I_{\delta}^{\prime}(z)$ near the gap. Let us first consider $I_{\delta}(z)$ and introduce $\alpha=k|z|, x=\beta|z|$ and $\gamma=\delta|z|^{-1}$ into (20) which becomes

$I_{\delta}(z)=2 i a \omega \epsilon \int_{0}^{\infty} \frac{\sin \gamma x \cos x}{\gamma x\left(\alpha^{2}-x^{2}\right)^{1 / 2}} \frac{H_{0}^{(2) \prime}\left(\left[\alpha^{2}-x^{2}\right]^{1 / 2} k a \alpha^{-1}\right)}{H_{0}^{(2)}\left(\left[\alpha^{2}-x^{2}\right]^{1 / 2} k a \alpha^{-1}\right)} d x$.

We wish to evaluate the integral in (24) for $0<\alpha<<1$. To this end we observe that if $c$ is any complex number the argument of the Hankel function in (24) has the value $-i c$ when $x=$ $\alpha\left[1+c^{2}(k a)^{-2}\right]^{1 / 2}$. Thus no matter how large the constant $c$ is, the absolute value of the argument of the Hankel function exceeds $c$ for all $x$ greater than a certain complex multiple of $\alpha$ which vanishes as $\alpha$ does. Consequently for all these values of $x$, the
Hankel functions in (24) differ by an arbitrarily small amount from their asymptotic forms. Furthermore the error resulting from replacing the Hankel functions by their asymptotic forms for smaller values of $x$ vanishes as $\alpha$ tends to zero. Thus we may write

$$
I_{\delta}(z)=2 a \omega \epsilon \int_{0}^{\infty} \frac{\sin \gamma x \cos x}{\gamma x\left(\alpha^{2}-x^{2}\right)^{1 / 2}} d x+0(\alpha) .
$$

Here we have used the asymptotic form

$$
\begin{aligned}
& H_{0}^{(2)}(-i y)=\frac{2 i}{\pi} K_{0}(y) \\
& \quad \sim i\left(\frac{2}{\pi y}\right)^{1 / 2} e^{-y}\left[1+\frac{\Gamma(3 / 2)}{2 y \Gamma(-1 / 2)}+0\left(y^{-2}\right)\right]
\end{aligned}
$$

In the preceding equations the branch of $\left(\alpha^{2}-x^{2}\right)^{1 / 2}$ employed is that which is equal to $-i\left|\left(\alpha^{2}-x^{2}\right)^{1 / 2}\right|$ when $x^{2}>\alpha^{2}$. From (26) it follows that the ratio of Hankel functions in (24) is just $-i$. In the same way we find that $I_{\delta}^{\prime}(z)$ is also given by the right side of $(25)$.

To evaluate the integral in (25) we introduce a division point $b$ and write it as two integrals. In the second integral we omit $\alpha^{2}$, introducing an error of order $\alpha^{2}$. Thus (25) becomes

$$
\begin{aligned}
I_{\delta}(z)=2 i a \omega \epsilon\left[\int_{0}^{b} \frac{\sin \gamma x \cos x}{\gamma x\left(x^{2}-\alpha^{2}\right)^{1 / 2}} d x\right. \\
\left.\quad+\int_{b}^{\infty} \frac{\sin \gamma x \cos x}{\gamma x^{2}} d x\right]+0(\alpha)
\end{aligned}
$$

We now integrate the first integral in (27) by parts, neglect terms of order $\alpha$, and obtain

$$
\begin{gathered}
I_{\delta}(z)=2 i a \omega \epsilon\left[-\log (-i \alpha)+\frac{\sin \gamma b}{\gamma b} \cos b \log 2 b\right. \\
\quad-\int_{0}^{b} \log 2 x \frac{d}{d x}\left(\frac{\sin \gamma x \cos x}{\gamma x}\right) d x \\
\left.\quad+\int_{b}^{\infty} \frac{\sin \gamma x \cos x}{\gamma x^{2}} d x\right]+0(\alpha) .
\end{gathered}
$$

The right side of (28) is obviously independent of $b$ so we set $b=1 / 2$, which makes $\log 2 b=0$. We also substitute $\alpha=k|z|$ and obtain finally

$$
\begin{gathered}
I_{\delta}(z)=-2 i a \omega \epsilon\left[\log k|z|-i \frac{\pi}{2}\right. \\
\quad+\int_{0}^{1 / 2} \log 2 x \frac{d}{d x}\left(\frac{\sin \gamma x}{\gamma x} \cos x\right) d x \\
\left.\quad-\int_{1 / 2}^{\infty} \frac{\sin \gamma x}{\gamma x^{2}} \cos x d x\right]+0(\alpha) .
\end{gathered}
$$

Here $\gamma=\delta|z|^{-1}$. The expression (29) for $I_{\delta}(z)$ is our result for the current near the gap.

When the gap width $\delta=0$ and $z \neq 0$ then $\gamma=$ $\delta|z|=0$ and $(29)$ becomes 


$$
\begin{array}{r}
I_{0}(z)=-2 i a \omega \epsilon\left[\log k|z|-i \frac{\pi}{2}-\int_{0}^{1 / 2} \log 2 x \sin x d x\right. \\
\left.-C i\left(\frac{1}{2}\right)\right]+0(\alpha) .
\end{array}
$$

The total current is $2 I_{0}(z)+0(\alpha)$. The term $-4 i a \omega \epsilon \log z$ in the total current for $\delta=0$ was given by Wu and King [1959], Duncan and Hinchey [1960], and G. Hasserjian and A. Ishimaru [1961]. The former two pairs of authors discussed an ambiguity in the current near the gap which is resolved by our additional terms in (30).

The input admittance $Y$ when $\delta>0$ is given by our result (29) evaluated at $z=\delta$.

$$
\begin{array}{r}
Y=-2 i a \omega \epsilon\left[\log k \delta-i \frac{\pi}{2}+\int_{0}^{1} \log y \frac{d}{d y}\left(\frac{\sin y}{y}\right) d y\right. \\
\left.-\int_{1}^{\infty} \frac{\sin y}{y^{2}} d y\right]+0(k \delta) .
\end{array}
$$

If currents on both sides of the pipe are excited, this result must be multiplied by two. The leading term in the admittance is imaginary and proportional to $\log k \delta$, which becomes infinite as $\delta$ vanishes. The next term, which is independent of $\delta$, contains the real part $-\pi a \omega \epsilon$ (or $-2 \pi a \omega \epsilon$ when currents are excited on both sides) which is the input conductance. Since it is independent of the gap width, it would presumably result for any type of antenna feed.

An incorrect result for the current at a gap of zero width was obtained by J. A. Stratton and L. J. Chu [1941]. Their error consisted in evaluating the integral for the current by replacing it by a sum of residues, ignoring the fact that the integrand contained a branch point. The integral should have been replaced by a branch cut integral plus the residue sum. As a consequence they concluded that the input admittance of the infinite antenna with a gap of zero width is zero, whereas it is actually infinite as our result (31) shows.

Another incorrect result for the current at a gap of zero width follows from the thin antenna expression (23) with $\delta=0$, obtained by Hallen [1956], Vladimirskii [1944], and Vainshtein [1959a, b]. The latter two authors showed that (23) yields

$$
I_{0}(0)=-\frac{1}{\sqrt{\frac{\epsilon}{\mu}} \log \Gamma k a}[1+0(1)] .
$$

This same result was obtained by Schelkunoff [1941], Hallen [1948], and $\mathrm{Wu}$ [1961]. The correct result (30) for $\delta=0$ is infinite at $z=0$ in contrast to the approximate expression (32) which is finite. The error in obtaining (32) from the exact expression (20) was made in interchanging the limiting processes $z \rightarrow 0$ and $k a \rightarrow 0$. The convergence to these limits is nonuniform. Ther efore, it is not permissible to first let $k a \rightarrow 0$, in which case (20) is asymptotic to (23), and then let $z \rightarrow 0$, when (23) approaches (32). The nonuniformity manifests itself in a small interval around the gap, which we may call a boundary layer. Within this layer the current drops from its large value at $z=\delta$ (or from its infinite value when $\delta=0$ ) to a small value. For example (30) shows that $I_{\delta}(z)=i \alpha$, where $\alpha$ is some small finite current, when $|z|=k^{-1} e^{-\alpha / z a \omega \epsilon}$. Therefore, the width of the boundary layer is very small when $k a$ is small. Within this layer the thin antenna theory is inapplicable. It is precisely in this layer where the current must be evaluated in order to determine the input admittance of the antenna.

\section{The Current Far From the Gap}

Let us now evaluate the current $I_{\delta}(z)$ far from the gap, starting from (20) which we write as

$$
I_{\delta}(z)=i a \omega \epsilon \int_{-\infty}^{\infty} \frac{\sin \beta \delta}{\beta \delta} \frac{H_{0}^{(2) \prime}\left(\left[k^{2}-\beta^{2}\right]^{\frac{1}{2}} a\right)}{H_{0}^{(2)}\left(\left[k^{2}-\beta^{2}\right]^{\frac{1}{2}} a\right)} \frac{e^{-i \beta|z|}}{\left[k^{2}-\beta^{2}\right]^{\frac{1}{2}}} d \beta .
$$

Since (33) expresses $I_{\delta}(z)$ as a Fourier integral, its value for large $|z|$ (actually large $k|z|$ ) is determined by the singularities of the integrand. These occur at $\beta= \pm k$ and therefore it suffices to replace the Hankel function in (33) by its asymptotic expansion for small values of its argument

$$
H_{0}^{(2)}\left(\left[k^{2}-\beta^{2}\right]^{\frac{1}{2}} a\right) \sim \frac{2 i}{\pi} \log \frac{2}{a \Gamma\left[k^{2}-\beta^{2}\right]^{\frac{1}{2}}} .
$$

Here $\Gamma \approx 1.781$ is Euler's constant. Since $\beta \delta= \pm k \delta$ at the singularities, and $k \delta$ is assumed to be small, we also replace $\sin \beta \delta / \beta \delta$ by unity. Then (23) becomes

$$
I_{\delta}(z) \sim i \omega \epsilon \int_{-\infty}^{\infty} \frac{e^{-i \beta|z|} d \beta}{\left(k^{2}-\beta^{2}\right) \log \frac{\Gamma a\left[k^{2}-\beta^{2}\right]^{1 / 2}}{2}} .
$$

We observe that when $\delta=0$ the thin antenna result (23) coincides with (35).

The integral in (35) has been evaluated asymptotically for $k z>>1$ by Vladimirskii [1944], Hallen [1948], Northover [1958], and Vainshtein [1959a, b] with the result

$$
I_{\delta}(z) \sim \frac{2 \pi \omega \epsilon}{k} \frac{e^{-i k|z|}}{\log \frac{2 i|z|}{\Gamma^{2} k a^{2}}} .
$$

This result also applies to thin antennas.

\section{Reflection From the End}

Let us now consider the reflection of the current from an end of a cylindrical antenna. This problem has been solved by E. Hallen [1956] for a hollow tube of circular cross section and for a thin antenna of any cross section. It has also been treated by L. A. Vainshtein [1959a, b] and T. T. Wu [1961]. They only complete their analysis to obtain explicit results for 
thin antennas. The antenna is assumed to be semiinfinite lying in the region $z>0$ with its end at $z=0$. The incident current is assumed to be $e^{i k z}$. For the hollow circular antenna of radius $a$ Hallen finds the reflected current on the outside of the antenna to be

$$
\begin{aligned}
I_{1}(z)= & \frac{+i k a}{2 \pi \phi(k a)} \\
& \int_{-\infty}^{\infty} \frac{e^{-i \beta z}}{\phi(\beta a)\left(k^{2}-\beta^{2}\right)^{1 / 2}} \frac{H_{0}^{(2) \prime}\left(\left[k^{2}-\beta^{2}\right]^{1 / 2} a\right)}{H_{0}^{(2)}\left(\left[k^{2}-\beta^{2}\right]^{1 / 2} a\right)} d \beta .
\end{aligned}
$$

Here $\phi(\alpha a)$ is defined by

$$
\begin{aligned}
& \phi(\alpha a)=\exp \frac{1}{2 \pi i} \int_{-\infty+i \epsilon}^{\infty+i \epsilon} \frac{d \zeta}{\zeta-\alpha} \\
& \log \left[\frac{\pi}{i} J_{0}\left(\sqrt{k^{2}-\zeta^{2}} a\right) H_{0}^{(2)}\left(\sqrt{k^{2}-\zeta^{2}} a\right)\right] .
\end{aligned}
$$

This result was obtained by using the Wiener-Hopf method to solve the integral equation satisfied by the total current on the cylinder, and then separating the current into its inner and outer parts.

If $|k a|<<1$, the cylinder functions in (37) and (38) can be replaced by the leading terms in their expansions for small values of their arguments, and they become

$$
I_{1}(z)=\frac{i k}{2 \pi \phi^{2}(k a)} \int_{-\infty}^{\infty} \frac{e^{-i \beta z} d \beta}{\left(k^{2}-\beta^{2}\right) \log \frac{\Gamma a \sqrt{k^{2}-\beta^{2}}}{2}}
$$

$\phi(\alpha a)=\exp \frac{1}{2 \pi i} \int_{-\infty+i \epsilon}^{\infty+i \epsilon} \frac{d \zeta}{\zeta-\alpha} \log \log \frac{-4}{a^{2} \Gamma^{2}\left(k^{2}-\zeta^{2}\right)}$.

The result (39) with $\phi$ given by (40) was also obtained by Hallen by solving his simplified integral equation for the current on a thin antenna. If the cross section is not circular, $a$ represents its effective radius.

Let us now evaluate $I_{1}(z)$ for large $|k z|$. Since $I_{1}(z)$ is expressed by (37) as a Fourier integral, the asymptotic behavior of it for large $|k z|$ is determined by the neighborhoods of the singularities of the integrand. The function $\phi(\alpha a)$ is regular and different from zero in the lower half of the $\alpha$ plane and the only singularity of the integrand of (37) in that plane is at $\alpha=k$. The singularity in the upper half plane at $z=-k$ is irrelevant in the evaluation of $I_{1}(z)$ for large positive values of $z$. Thus we may replace $\phi(\alpha a)$ by $\phi(k a)$ in (37) which then becomes, upon comparison with (23)

$$
I_{1}(z) \sim \frac{k}{2 \pi \omega \epsilon \phi^{2}(k a)} I_{\delta}(z) .
$$

Thus our assumption (9) that $I_{1}(z)$ is proportional to $I_{\delta}(z)$ for large $z$ is verified. The proportionality constant $C$ in (9) is seen to be, from (41),

$$
C=\frac{k}{2 \pi \omega \epsilon \phi^{2}(k a)} .
$$

The results (41) and (42) also apply to the thin antenna with $\phi$ given by (40).
Twice the reciprocal of the constant $C$ occurs in the work of Hallen and he calls it the end admittance of the antenna. He has computed its real and imaginary parts for a large range of $k a$, assuming $\sigma=0$, using both (38) and (40) for $\phi(k a)$. The two resulting values of $C$ are practically equal for $k a<0.1$. For small values of $k a$

and

$$
\phi^{2}(k a)=\frac{-1}{2 \log i \Gamma k a}+0\left[\frac{1}{(\log k a)^{3}}\right]
$$

$$
C=\frac{-k}{\pi \omega \epsilon}\left[\log i k a \Gamma+0\left(\frac{1}{\log k a}\right)\right] \text {. }
$$

For the thin antenna it follows from (39) and (40) that $I_{1}(0)=-1$ so that the incident plus the reflected current is zero at the end. This verifies the assumption usually made in the theory of thin antennas.

\section{The Finite Cylindrical Antenna}

Now $I_{\delta}(z), I_{1}(z)$, and $C$ have been determined for cylindrical antennas of circular cross section and for thin cylindrical antennas of any cross section. Therefore we may use them in the formulas of section 2 to obtain the current on and input admittance of the corresponding finite cylindrical antennas. Thus when $z$ is not near the gap nor the ends, (4) becomes

$$
\begin{gathered}
I(z)=\frac{2 \pi \omega \epsilon}{k}\left\{\frac{e^{-i k|z|}}{\log \alpha|z|}+\frac{1}{\phi^{2}(k a)-\frac{1}{\phi^{2}(k a)} \frac{e^{-i 2 k\left(z_{1}+z_{2}\right)}}{\left[\log \alpha\left(z_{1}+z_{2}\right)\right]^{2}}}\right. \\
\cdot\left[\frac { e ^ { - i k ( z + 2 z _ { 1 } ) } } { \operatorname { l o g } \alpha ( z _ { 1 } + z ) } \left(\frac{1}{\log \alpha z_{1}}\right.\right. \\
\left.+\frac{e^{-i k 2 z_{2}}}{\phi^{2}(k a) \log \alpha z_{2} \log \alpha\left(z_{1}+z_{2}\right)}\right)+\frac{e^{-i k\left(2 z_{2}-z\right)}}{\log \alpha\left(z_{2}-z\right)}\left(\frac{1}{\log \alpha z_{2}}\right. \\
\left.\left.\left.+\frac{e^{-i 2 k z_{1}}}{\phi^{2}(k a) \log \alpha z_{1} \log \alpha\left(z_{1}+z_{2}\right)}\right)\right]\right\} . \quad(45)
\end{gathered}
$$

Here $\alpha=2 i / \Gamma^{2} a^{2} k$. The input admittance $Y$ becomes

$$
\begin{aligned}
& Y=-2 i a \omega \epsilon\left[\log k \delta-i \frac{\pi}{2}+\int_{0}^{1} \log y \frac{d}{d y}\left(\frac{\sin y}{y}\right) d y\right. \\
& \left.-\int_{1}^{\infty} \frac{\sin y}{y^{2}} d y\right]+\frac{2 \pi \omega \epsilon}{k}\left\{\frac{1}{\phi^{2}(k a)-\frac{e^{-i 2 k\left(z_{1}+z_{2}\right)}}{\phi^{2}(k a)\left[\log \alpha\left(z_{1}+z_{2}\right)\right]^{2}}}\right. \\
& {\left[\frac{e^{-i 2 k z_{1}}}{\left(\log \alpha z_{1}\right)^{2}}+\frac{e^{-i 2 k z_{2}}}{\left(\log \alpha z_{2}\right)^{2}}\right.}
\end{aligned}
$$

$$
\left.\left.+\frac{2 e^{-i 2 k\left(z_{1}+z_{2}\right)}}{\phi^{2}(k a) \log \alpha z_{1} \log \alpha z_{2} \log \alpha\left(z_{1}+z_{2}\right)}\right]\right\} \text {. }
$$


For the center fed antenna, these results simplify to

$$
\begin{aligned}
I(z)= & \frac{2 \pi \omega \epsilon}{k}\left\{\frac{e^{-i k|z|}}{\log \alpha|z|}\right. \\
+ & \left.\frac{\left[\frac{e^{-i k z}}{\log \alpha\left(z_{1}+z\right)}+\frac{e^{i k z}}{\log \alpha\left(z_{1}-z\right)}\right] \log 2 \alpha z_{1}}{\log \left[\phi^{2}(k a)\left(\log 2 \alpha z_{1}\right) e^{i 2 k z_{1}}-1\right]}\right\} \\
Y=-2 i a \omega \epsilon & {\left[\log k \delta-i \frac{\pi}{2}\right.} \\
+ & \left.\int_{0}^{1} \log y \frac{d}{d y} \frac{\sin y}{y} d y-\int_{1}^{\infty} \frac{\sin y}{y^{2}} d y\right] \\
& +\frac{4 \pi \omega \epsilon \log 2 \alpha z_{1}}{k\left(\log \alpha z_{1}\right)^{2}\left[\phi^{2}(k a) e^{i 2 k z_{1}} \log 2 \alpha z_{1}-1\right]} .
\end{aligned}
$$

For thin antennas, when $C$ is given by (44), the results (45) and (47) are the same as those given by Vainshtein [1959a, b].

\section{References}

Duncan, R. H., and F. A. Hinchey, Cylindrical antenna theory, J. Research NBS 64D (Radio Prop.), 569-584 $(1960)$.
Hallen, E., Properties of a long antenna, J. Appl. Phys. 19, 1140-1147 (1948).

Hallen, E., Exact treatment of current wave reflection at the end of a tube-shaped cylindrical antenna, IRE Trans. on Ant. Prop. AP-4, 479-491 (1956).

Hasserjian, G., and A. Ishimaru, Currents induced on circular cylinders by slots, URSI-IRE meeting, Washington, D.C. (May 1961).

King, R. W. P., Theory of linear antennas (Harvard Univ. Press, Cambridge, Mass., 1956).

Northover, F. H., Radiation and surface currents from a slot on an infinite conducting cylinder, Can. J. Phys. 36, 206-217 (1958).

Schelkunoff, S. A., Theory of antennas of arbitrary size and shape, Proc. IRE 29, 493-521 (1941).

Stratton, J. A., and L. J. Chu, Steady state solutions of electromagnetic field problems. I. Forced oscillations of a cylindrical conductor, J. Appl. Phys. 12, 231-235 (1941).

Vainshtein, L. A., Waves of current in a thin cylindrical conductor. I. Currents and impedance of a transmitting antenna, J. Tech. Phys, USSR 99, No 6 (1959); English translation: Soviet Phys. Tech. Phys. 4, 601-615 (1959a).

Vainshtein, L. A., Waves of current in a thin cylindrical conductor. II. The current in a passive oscillator and the radiation of a transmitting antenna, ibid. 617-626 (1959b)

Vladimirskii, V. A., Izvest. Akad. Nauk SSSR, Ser. Fiz 8, 139-149 (1944).

$\mathrm{Wu}, \mathrm{T}$. T., Theory of the dipole antenna and the two wire transmission line, J. Math. Phys. 2, 550-574 (1961).

Wu, T. T., and R. W. P. King, Driving point and input admittance of linear antennas, J. Appl. Phys. 30, 74-76 (1959). 